www.jmscr.igmpublication.org

Impact Factor 5.84

Index Copernicus Value: 83.27

ISSN (e)-2347-176x ISSN (p) 2455-0450

crossref DOI:_https://dx.doi.org/10.18535/jmscr/v5i7.01

\title{
Receive Bandwidth Variation Effect Toward the Signal and Contrast to Noise Ratio in Magnetic Resonance Imaging (MRI) Cervical Examination with the Sequence of T1 Weighted Turbo Spin Echo
}

\author{
Authors

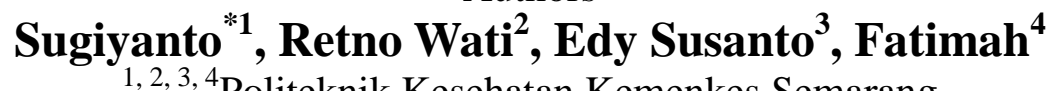 \\ 1, 2, 3, ${ }^{4}$ Politeknik Kesehatan Kemenkes Semarang \\ Jl. Tirto Agung Pedalangan, Banyumanik, Semarang 50239, Indonesia \\ Corresponding Author \\ Sugiyanto \\ Email:iskakgie@gmail.com
}

\begin{abstract}
Introduction: The received bandwidth parameter is a parameter affecting the value of Signal to Noise Ratio (SNR) where the radiographer is usually stuck to the existing protocol: the bandwidth of $146 \mathrm{~Hz} / \mathrm{Px}$. In fact, by doing the variation of received bandwidth, the pictures of SNR variation will be obtained to define the best bandwidth to SNR and contrast to noise ratio (CNR).

Objective: The purpose of this research is to find out the effect of received bandwidth variation on SNR and CNR and to determine the most optimal received bandwidth for SNR and CNR on cervical MRI with the sequence of T1 Weighted Turbo Spin Echo (TSE) in the sagittal slice.

Methods: The type of this research is quantitative research with experimental approach done with MRI 1.5 Tesla at General Hospital Dr. Hasan Sadikin Bandung. The data were 35 cervical TRL images of TSE in the sagittal slice from 7 probands with 5 received bandwidth variations (106 Hz / Px, $126 \mathrm{~Hz} / \mathrm{Px}, 146 \mathrm{~Hz} / \mathrm{Px}$, $166 \mathrm{~Hz} / \mathrm{Px}$ and $186 \mathrm{~Hz} / \mathrm{Px}$ ). To obtain SNR and CNR values, an area of interest (ROI) measurement is performed on the corpus, discus, spinal cord and CSF regions to obtain the average signal and compared with the standard deviation value of the background. Data was analyzed with linear regression and descriptive mean rank test.

Result: The result of the research shows the effect of received bandwidth on SNR and CNR on MRI cervical. There is a strong correlation between received bandwidth variation with cervical SNR with correlation coefficient $=0.639$, and there is a moderate correlation between received bandwidth variation with CNR cervical with correlation coefficient $=0,532$ with $p$-value $<0.05$. The optimal received bandwidth value of the T1WI saturation T1WI TIWI MRI is $106 \mathrm{Hx} / \mathrm{px}$ with a mean rank of SNR $=160.5$ and mean rank of $C N R=76.59$.

Conclusion: The received bandwidth variations on SNR and CNR in MRI significantly affect the cervical examination. The optimal received bandwidth value of T1WI TSE in the sagittal slice is $106 \mathrm{Hx} / \mathrm{px}$.

Keywords: received bandwidth, SNR, CNR, MRI cervical, T1 weighted TSE.
\end{abstract}




\section{INTRODUCTION}

Diagnostic radio examination is one of the investigations in the field of medicine in helping diagnose a disease. One of the diagnostic radio checks is an examination using the imaging modalities of Magnetic Resonance Imaging (MRI). MRI examination produces images of human body pieces using a magnetic field without using X-rays. Examination using MRI is capable of producing better images and has several advantages such as providing good spatial resolution, good inter-tissue contrast without ionizing radiation and produces images with various pieces (multi-planar) such as axial, coronal and sagittal pieces without reconstruction of the image first (Rasad, 2011).

According to Wesbrook \& Catherine, (2002), there are many sequencing techniques on MRI examination, one of the frequently used sequencing techniques is Turbo Spin Echo (TSE). TSE has the advantage that the acquisition time is faster because there are Echo Train Length (ETL) parameters that are active in this sequence making TSE is used almost 40-60\% in MRI examination.

Spine MRI examination is an examination that is often found in the field including a cervical MRI examination. The cervical vertebrae are unique, because the corpus is smaller than the thoracal and lumbar vertebrae, and also has a crane base joint. Movements in this object are more due to the process of swallowing. In general, the sagittal piece will be able to provide a more thorough visualization, especially an assessment of the spinal cord, discus inter-vertebra, soft tissue of the neck, the lateral aspect of the corpus and the inferior aspect of the posterior cranial fossa (Woodward, 1997).

The optimal image quality of MRI is determined by four characteristics, namely signal to noise ratio (SNR), contrast to noise ratio (CNR), spatial resolution and scan time. Each signal obtained by each voxel element will be measured in the MRI equipment into a signal to noise ratio (SNR) and affect the contrast to noise ratio (CNR) (Westbrook, et.al 1998).
Many parameters affect the MRI scanning that can be controlled directly and indirectly by a radiographer. As a radiographer, it is important to know how the inspection runs and how to manage the many parameters in MRI to produce an optimum image quality.

Parameter settings will affect the SNR and CNR values where one of them is received bandwidth. Received bandwidth is the range of frequencies used for data acquisition. The bandwidth width is determined by the gradient read-out power and the data sampling rate that specifically affects the MRI system. Received bandwidth does not affect signal strength, but is closely related to the amount of noise (Westbrook et.al, 1998). There are many variations of received bandwidth on each type of MRI plane and to know the value of optimal received bandwidth on an MRI plane then it is necessary to do an experiment. According to Sundseth (2013) on magnetic field system, 1.5 Tesla for MRI cervical sequence T1 TSE sequence in the sagittal cutoff, the received bandwidth used is $\pm 150 \mathrm{~Hz} / \mathrm{Px}$. However, the author's observation in some hospitals received bandwidth ranges used are varied from $186 \mathrm{~Hz} / \mathrm{Px}, 156 \mathrm{~Hz}$ / Px, and 146Hz / px.

Based on the author's observations during field practice, usually, the radiographer is stuck to the parameters of the existing protocol where on MRI cervical examination, in General Hospital Dr.Hasan Sadikin Bandung, T1WI TSE with the bandwidth range of $146 \mathrm{Hx} / \mathrm{px}$ is commonly used. The received bandwidth variations are 106 Hz / Px, 126 Hz / Px, 146 Hz / Px, 166 Hz / Px and $186 \mathrm{~Hz} / \mathrm{Px}$ respectively. According to Westbrook (1998) bandwidth can affect SNR, but it has not been done statistically so research needs to be done. The purpose of this research is to know the effect of receive bandwidth variation on signal to noise ratio (SNR) and contrast to noise ratio (CNR) on cervical MRI examination with $\mathrm{T} 1$ Weighted Turbo Spin Echo sequence as well as to determine the value of optimal receive bandwidth on cervical MRI examination with T1 Weighted Turbo Spin Echo sequence. 


\section{MATERIALS AND METHODS}

The type of this research is quantitative research with experimental approach. The data were collected from May to June 2016 at Radiology Installation of Dr. Hasan Sadikin Bandung Hospital. The population and sample of the study were MRI cervical sequences of T1 Weighted Turbo Spin Echo (TSE) sagittal pieces using 5 received bandwidth variations from 7 probands. The research procedures conducted by performing a MRI cervical sequence of T1WI TSE sequences with sagittal slice by adjusting the received bandwidth parameters found in the tool with variation of received bandwidth at $106 \mathrm{~Hz} / \mathrm{Px}$, $126 \mathrm{~Hz} / \mathrm{Px}, 146 \mathrm{~Hz} / \mathrm{Px}, 166 \mathrm{~Hz} / \mathrm{Px}$ and $186 \mathrm{~Hz}$ / Px while other parameters remained fixed. Then the image results in the mid-cervical area are evaluated by measuring the SNR and CNR values in the area to be evaluated and the free area to find out the average of each image signal. SNR measurement is done by ROI as small as possible $\pm 1 \mathrm{~mm}$ on monitor in corpus, discus, spinal cord and CSF areas. CNR measurement is done by calculating the difference of SNR value of the two networks. CNR in this study is the difference between SNR corpus with CSF, discus with CSF, spinal cord with CSF, spinal cord with corpus, spinal cord with disc and corpus with disc.

Data analysis was done with the aids of SPPS 16 for Windows application. The data were tested with the Shapiro-Wilk test to determine the normality of the data. Further data is processed by linear regression test to know whether there is a significant effect of received bandwidth variation on SNR and CNR on MRI cervical inspection T1 Weighted Turbo Spin Echo in sagittal slices. The descriptive mean rank test is performed to determine the variation of the optimal bandwidth gain.

\section{RESULTS AND DISCUSSION}

This study was conducted on 7 male and female probands, aged from 19 years to 24 years old whose weight $45 \mathrm{~kg}$ up to $65 \mathrm{~kg}$ undergone cervical MRI examination with T1WI TSE sequence of sagittal pieces with 5 received bandwidth variation of $106 \mathrm{~Hz} / \mathrm{Px}, 126 \mathrm{~Hz} / \mathrm{Px}$, $146 \mathrm{~Hz} / \mathrm{Px}, 166 \mathrm{~Hz} / \mathrm{Px}$ and $186 \mathrm{~Hz} / \mathrm{Px}$.

Table 1. Probands' Characteristics

\begin{tabular}{c|c|c|}
\hline Probands & Ages/Gender & Weight \\
\hline 1 & 24 years/man & $65 \mathrm{kgs}$ \\
2 & 19 years/man & $63 \mathrm{kgs}$ \\
3 & 20 years/man & $60 \mathrm{kgs}$ \\
4 & 21 years/ woman & $55 \mathrm{kgs}$ \\
5 & 22 years/ woman & $48 \mathrm{kgs}$ \\
6 & 24 years/ woman & $58 \mathrm{kgs}$ \\
7 & 22 years/woman & $45 \mathrm{kgs}$ \\
\hline
\end{tabular}

From the seven probands were obtained the SNR and CNR value from received bandwidth variation on cervical MRI examination with T1WI TSE sequence of sagittal pieces as presented in Figure 1.

Figure 1. The image of received bandwidth variation of $106 \mathrm{~Hz} / \mathrm{px}, 126 \mathrm{~Hz} / \mathrm{px}, 146 \mathrm{~Hz}$ / px, $166 \mathrm{~Hz} / \mathrm{px}$ and $186 \mathrm{~Hz} / \mathrm{px}$.

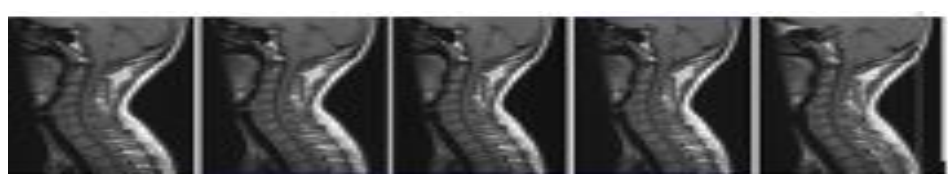

\section{Effect of Received Bandwidth variation on SNR and CNR on MRI Cervical Examination}

Image assessment is done with the area of interest (ROI) and measuring SNR of each image: SNR corpus, discus, spinal cord, and CSF. After SNR calculation, CNR calculation is continued which is variation of the adjacent SNR in one image. Calculated are CNR corpus with CSF, discus with CSF, spinal cord with CSF, spinal cord with corpus, spinal cord with disc and corpus with disc. SNR and CNR data obtained, before the linear regression test is done, normality data test by using Shapiro-Wilk test was conducted to know whether the data is normally distributed. Based on the data normality test results, then the data are obtained in normal distribution with the $\mathrm{p}$ - value $>0.05$.

The result of regression test to reveal the influence between the various received bandwidth to SNR value on T1WI TSE MRI cervical is presented in Table 2. 
Table 2. Regression test on received bandwidth to SNR

\begin{tabular}{|l|c|c|c|}
\hline SNR & $\mathrm{R}$ & $R$ Square & $p$-Value \\
\hline Cervical & 0.639 & 0.408 & $<0.05$ \\
\hline
\end{tabular}

The table indicates that there is a strong correlation between the variation of received bandwidth to cervical SNR withcorrelation coefficient $\mathrm{r}=0.639, \mathrm{p}$ - value $<0.05$, and coefficient of determination ( $\mathrm{R}$ Square) $=0.408$ meaning that $40.8 \%$ of SNR is influenced by received bandwidth while $59.2 \%$ of SNR is influenced by other factors.

To determine the result of received bandwidth variation to SNR in every organ: SNR in corpus, discus, spinal cord and CSF, regression test is performed with the following results:

Table 3. Regression test results on received bandwidth to SNR per organ

\begin{tabular}{|l|c|c|c|l|}
\hline SNR & $\mathrm{R}$ & $R$ Square & $p$-Value & Conclusion \\
\hline Corpus & 0.733 & 0.537 & $<0.05$ & Significant \\
\hline Discus & 0.401 & 0.161 & $<0.05$ & Significant \\
\hline Spinal Cord & 0.638 & 0.407 & $<0.05$ & Significant \\
\hline CSF & 0.423 & 0.179 & $<0.05$ & Significant \\
\hline
\end{tabular}

The table shows that there is a strong correlation between variation of received bandwidth to SNR corpus, with correlation coefficient $=0.733$ and coefficient of determination $=0,537$ indicating that $53.7 \%$ of SNR is influenced by received bandwidth while $46.3 \%$ influenced by other factors. In contrast, there is a weak correlation between the variation of received bandwidth with respect to SNR discus with correlation coefficient $=0.401$ and coefficient of determination $=0.161$ meaning that $16.1 \%$ of SNR is influenced by received bandwidth while $83.9 \%$ influenced by other factors. Furthermore, there is a strong correlation between the variation of received bandwidth to SNR spinal cord with correlation coefficient $=0.638$ and coefficient of determination $=0.407$ meaning $40.7 \% \mathrm{SNR}$ is influenced by received bandwidth while $59.3 \%$ influenced by other factors. Finally, there is a weak correlation between the variation of receive bandwidth to SNR CSF with correlation coefficient $=0.423$ coefficient of determination $=$ 0.179 which means only $17.9 \%$ of SNR is influenced by received bandwidth while $82.1 \%$ is influenced by other factors.

Based on these results it is concluded that the value of received bandwidth affects SNR which is the ratio between the magnitude of the amplitude of the signal with the amplitude of noise. According to Hashemi (1997), when bandwidth is narrower, SNR will increase. Received bandwidth is the frequency range that occurs in sampling data on the object being scanned. The smaller the bandwidth the noise will be smaller. According to Blink (2004), received smaller bandwidth can be interpreted as slower sampling as it takes longer time to collect the same number of points in the data. Based on these results then this research is in accordance with the existing theory where the smaller received bandwidth will increase the SNR. The affecting factor is that with the small receive bandwidth, the Field of View (FOV) becomes smaller as it produces less noise. Because noise is an unwanted signal, where the signal is generated by a superposition signal with the actual image. Noise exists at all frequencies, if the measured frequency range is large then more noise will contribute to the signal. This is because the average pixel noise value is more dominant than the average pixel value of the actual signal. With a small received bandwidth producing a more smooth matrix, it will reduce the unwanted noise.

Other factors affecting SNR in addition to receiving bandwidth are network inherence (object), MRI component, and technical factors. Inherent tissue factors such as proton density of the examined area, where the higher the proton density the higher the resulting SNR value, the short relaxation time $\mathrm{T} 1$ and $\mathrm{T} 2$ cause the SNR to increase, the presence of physiological movements such as blood flow, CSF and chemical 
shift on the object causes SNR to decrease. MRI component such as main magnets, shim coils, radio frequency coils and computer systems if not well maintained and calibrated, will cause magnetic field strength and homogeneity level decrease hat signal decreases and noise increases (Westbrook \& Catherine, 2002).

Upon completion of SNR calculation, CNR calculation which is the adjacent SNR difference in one image is performed in the areas of CNR corpus with CSF, discus with CSF, spinal cord with CSF, spinal cord with corpus, spinal cord with discus, and corpus with discus.

The result indicates a moderate correlation between the variation of received bandwidth to CNR cervical with $\mathrm{p}$ - value $=<0.05$, correlation coefficient $r=0.532$ with coefficient of determination $=0.283$ meaning $28.3 \%$ of $\mathrm{CNR}$ is influenced by received bandwidth while $71.7 \%$ influenced by other factors.

There is a low correlation between the variation of received bandwidth to CNR corpus with CSF under the correlation coefficient 0.355 with coefficient of determination $=0.126$ meaning $12.6 \%$ of CNR is influenced by received bandwidth while $87.4 \%$ is influenced by other factors. There is a low correlation between the variation of received bandwidth to CNR disc with CSF under correlation coefficient $=0,263$ and coefficient of determination $=0.069$ meaning only $6.9 \%$ of CNR is influenced by received bandwidth while $93.1 \%$ is influenced by other factors. Similarly, there is a very low correlation between the variation of received bandwidth to CNR spinal cord with CSF under correlation coefficient $=0.180$ and coefficient of determination $=0.032$ indicating only $3.2 \%$ of CNR is influenced by received bandwidth while $96.8 \%$ influenced by other factors. There is a low correlation between the variation of received bandwidth to CNR spinal cord with corpus under correlation coefficient $=0.361$ and coefficient of determination $=0.130$ meaning $13 \%$ of CNR is influenced by received bandwidth while $87 \%$ is influenced by other factors. The consistent result is also found in a low correlation between the variation of received bandwidth to CNR spinal cord with discus under correlation coefficient 0.157 and coefficient of determination $=0.025$ indicating $2.5 \%$ of $\mathrm{CNR}$ is influenced by received bandwidth while $97.5 \%$ is influenced by other factors. Finally, a low correlation again is found in the variation of received bandwidth to CNR corpus with discus, under correlation coefficient $=0.358$ and coefficient of determination $=0.128$ confirming $12.8 \%$ of $\mathrm{CNR}$ is influenced by received bandwidth while $87 \%$ influenced by other factors. All correlation are significant for the p-value in calculation is also below 0.05 as in SNR calculation.

Based on the result of research it is concluded that the value of received bandwidth affects CNR though the correlation is only moderate to low.

Other factors affecting CNR in addition to received bandwidth are contrast media used such as using T2 sequence weighting, selecting magnetization transfer and eliminating normal network picture with spectral presaturation or using Short Tau Inversion Recovery (STIR) and Fluid Attenuated Inversion Recovery (FLAIR) for pressing a particular network (Westbrook \& Catherine, 2000).

\section{The most optimal Receive Bandwidth to generate Signal To Noise Ratio (SNR) and Contrast To Noise Ratio (CNR)}

Based on the calculation of SNR mean rank result, it can be seen that the received bandwidth variation of $106 \mathrm{~Hz} / \mathrm{px}$ has the highest SNR value that is 160.51 making received bandwidth of $106 \mathrm{~Hz} / \mathrm{px}$ produces the best SNR. The calculation of the result of mean SNR rank indicates that received bandwidth of $106 \mathrm{~Hz} / \mathrm{px}$ $=160.51,126 \mathrm{~Hz} / \mathrm{px}=141.05,146 \mathrm{~Hz} / \mathrm{px}=$ $121.51,166 \mathrm{~Hz} / \mathrm{px}=117.11$, and $186 \mathrm{~Hz} / \mathrm{px}=$ 111.00. From the calculation of the CNR mean rank it can be seen that the received bandwidth variation of $106 \mathrm{~Hz} / \mathrm{px}$ has the highest CNR value of 76.59 so the received bandwidth of 106 $\mathrm{Hz} / \mathrm{px}$ produces the best CNR. From the 
descriptive mean mean rank of CNR cervical, the result indicates that the received bandwidth of $106 \mathrm{~Hz} / \mathrm{px}=76.59,126 \mathrm{~Hz} / \mathrm{px}=58.84,146 \mathrm{~Hz}$ / $\mathrm{px}=56.40,166 \mathrm{~Hz} / \mathrm{px}=55.02$, and $186 \mathrm{~Hz}$ / $\mathrm{px}=53.00$.

In this study the smaller the received bandwidth then the greater the value of SNR and CNR but longer scanning time is required. The scanning time indicates that received bandwidth of 106 $\mathrm{Hz} / \mathrm{px}=02.26$ minutes, $126 \mathrm{~Hz} / \mathrm{px}=02.13$ minutes, $146 \mathrm{~Hz} / \mathrm{px}=02.07$ minutes, $\mathrm{Hz} / \mathrm{px}$ $=02.03$ minutes and $186 \mathrm{~Hz} / \mathrm{px}=2: 00$ minutes.

SNR and CNR are important to image quality criteria that can be used to produce good images with little contrast or for images with shorter scan times, higher resolutions, and fewer artifacts. Based on research conducted by the writers, the ideal SNR and CNR value occurs on received bandwidth with the value of $106 \mathrm{~Hz} / \mathrm{Px}$ with a scanning time of 02.26 minutes. Thus, to get the optimal SNR and CNR value on the cervical MRI examination with the sagittal piece does not require too long scanning time with the highest SNR and CNR values.

\section{CONCLUSIONS}

Received bandwidth variations significantly affect Signal to Noise Ratio (SNR) and Contrast to Noise Ratio (CNR) on the cervical MRI examination with T1 Weighted Turbo Spin Echo sequence with sagittal slice. A strong correlation exists between the variation of received bandwidth to cervical SNR proved with coefficient of correlation $=0.639$ and coefficient of determination $=0.408$ indicating $40.8 \%$ of SNR is influenced by received bandwidth while $59.2 \%$ of SNR influenced by other factors not included in the model. However, not like to SNR, only a moderate correlation exists between the variation of received bandwidth to CNR cervical with coefficient of correlation $=0.532$ and coefficient of determination $=0,283$ indicating that only $28.3 \%$ of CNR is affected by received bandwidth while $71.7 \%$ is influenced by other factors.
This research reveals that the value of received bandwidth variations to produce the most optimal SNR and CNR on cervical MRI with T1 Weighted Turbo Spin Echo sequence is at bandwidth of 106 $\mathrm{Hz} / \mathrm{Px}$. This way, in creating the cervical MRI images with T1 Weighted Turbo Spin Echo sequences, using received bandwidth of $106 \mathrm{~Hz} /$ Px as is preferable as it produces the optimal result. However, Ii is necessary to confirm the respondents (radiologist specialists) to assess whether high SNR and CNR values can provide optimal image information.

\section{REFERENCES}

1. Blink, Evert J. 2004. Basic MRI Physics. Application Specialist MRI

2. Hasemi, Ray, H., dan Bradley William G., 1997, MRI the Basic, Philadelphia: Lippicontt Williams \& Wilkins

3. Rasad, Sjahriar. 2011. Radiology Diagnostik, Edisi Kedua. Gaya Baru Balai Penerbit FKUI: Jakarta

4. Westbrook, Catherine, and Kaut, Carolyne. 1998. MRI in Practice, Second Edition. Blackwell Science Ltd: United Kingdom

5. Westbrook, Catherine. 2000. Handbook of MRI Technique, Second Edition. London: Blackwell Science

6. Westbrook, Catherine. 2002. MRI at Glance, United Kingdom: Blackwell Science

7. Woodward, Peggy and William W. Orrison. 1997. MRI Optimization; A Hands-on Approach. Mc. Graw Hill: New York, USA. 